but on this occasion heating was continued until the tube burst. The explosion took place in a small room, and we were able to show that an aerosol of potassium chromate had been formed. Most of the particles in a cloud sample, obtained by means of a thermal precipitator, were of the order of $1 \mu$ diameter, but a small number of more massive particles (about $10 \mu$ diameter) were also found. Although this cloud compared unfavourably, as a dispersion, with the smokes that can be made by conventional methods, we are of the opinion that a more uniformly and more finely dispersed system could be obtained by more careful control of the expansion and dilution of the vapour mixture than was attempted in the test we have described.

These observations were made in 1941, and we are grateful to the Director-General of Research (Defence), Ministry of Supply, for permission to publish this account. One of us (B.A.T.) is no longer in the service of the Ministry.

W. H. WALTON

B. A. Toms

Ministry of Supply,

Chemical Defence Experimental Station, Porton, Wilts.

Dec. 11.

${ }^{1}$ For a bibliography of earlier literature, see "Solubility Measurements in the Region of the Critical Temperature", by C. H. Holder and O. Maass, Canad. J. Res., 18, Sec. B, 293 (1940).

\section{Absolute Axes and Tidal Forces}

Ir has been shown ${ }^{1}$ that at any point of space-time, whether empty or occupied by matter, it is possible to define a system of absolute axial directions, independently of the Ricei tensor, and, in general, uniquely. The method makes use of a tensor which has since been proved by Ruse $^{2}$ to be identical with the conformal curvature tensor of Weyl. It may be supposed that this system of axes is of mathematical interest only, but it appears possible to give it a simple physical interpretation.

The complete Riemann - Christoffel tensor $B_{i j k l}$ cen be expressed in terms of the Ricei tensor $G_{i j}$, and the conformal curvature tensor $C_{i j k l}$ by the formula ${ }^{1,2}$ $B_{i j k l}=C_{i j k l}-\frac{1}{2}\left(g_{i k} G_{j l}+g_{j l} G_{i k}-g_{i l} G_{j k}-g_{j k} G_{i l}\right)+$

$$
\frac{1}{6}\left(g_{i k} g_{j l}-g_{i l} g_{j k}\right) G \text {. }
$$

Since $G_{i j}$ and $C_{i j k l}$ have each ten, and $B_{i j k l}$ has twenty, independent components, it follows that $G_{i j}$ and $C_{i j k l}$ are algebraically independent.

Thus, the conformal curvature tensor represents certain properties of the field which are independent of the Ricci tensor and, therefore, of the stress-andenergy tensor derived from it. These properties must be independent of the nature and motion of the matter at the point, since the latter are fully described by the stress-and-energy tensor. They must, therefore, represent effects of the outside world on conditions at the point.

The idea, therefore, suggests itself that the conformal curvature tensor represents the tidal forces, these forces being due to external gravitating bodies and showing themselves in a distortion of the shape of a deformable body placed at the point. That this interpretation is probable may be shown by considering a weak static field, which can be represented by a Newtonian potential.

Thus, in the Schwarzschild field of an isolated particle, the components of $C_{i j k l}$ reduce effectively to two, which may be written ${ }^{1}$
$C_{23}^{23}=C_{14}^{14}=-\frac{2 m}{r^{3}}, C_{31}^{31}=C_{24}^{24}=C_{12}^{12}=C_{34}^{34}=\frac{m}{r^{3}}$.

The Newtonian potential is

$$
V=\frac{m}{r}
$$

and we have

$$
C_{23}^{23}=-\frac{\partial^{2} V}{\partial x_{1}^{2}}, C_{31}^{s 1}=-\frac{\partial^{2} V}{\partial x_{2}^{2}}, C_{12}^{12}=-\frac{\partial^{2} V}{\partial x_{3}^{2}} ;
$$

$x_{1}, x_{2}, x_{3}$ being Cartesian co-ordinates coinciding locally with the spherical polars.

In this case, therefore, the solid harmonic of the second order which represents the disturbing potential of the tidal field is given by the quadratic form

$$
C_{23}^{23} d x_{1}^{2}+C_{31}^{31} d x_{2}^{2}+C_{12}^{x_{2}} d x_{3}^{2} \text {. }
$$

By superposition we can generalize this form for all Newtonian fields. To obtain a tensor form we must replace the linear elements $d x_{1}, d x_{2}, d x_{3}$ by the orthogonal surface-elements $d S^{23}, d S^{31}, d S^{12}$. We are thus led to consider the quadratic form

$$
C_{i j k l} d S^{i j} d S k l \text {, }
$$

which is the basis of the system of absolute axes referred to ${ }^{1}$

In a general Newtonian field the tidal forces at any point may be explored by placing there a small, freely falling, non-rotating fluid planet. This will then, in general, take up an ellipsoidal shape, under the joint influence of its own gravitation and the external tidal forces. The directions of the principal axes of the ellipsoid depend only on the external field and may be taken as the directions of a system of absolute local Cartesian co-ordinates.

In a general Einstein field, which may be strong and rapidly changing, the appropriate generalization of this system is given by the system of local Galilean axes with directions defined by the con. formal curvature tensor.

Admiralty Signal Establishment,

$$
\text { A. G. D. WATSON }
$$

\section{Haslemere,} Surrey.

1 Watson, A. G. D., Proc. Edin. Math. Soc. (2), 6, 12 (1939-41) 2 Ruse, H. S., Proc. Edin. Math. Soc., (2), 7, 144 (1942-45).

\section{Stabilization of Penicillin in Aqueous Solutions by Low Concentrations of Phosphates}

THE practical problems presented by the instability of penicillin in aqueous solution are well known, and much attention is being focused on their solution. The importance of these problems is intensified by the current trend toward commercial production of penicillins of increased purity, since it is now well recognized that, under comparable conditions of storage, sterile aqueous solutions prepared from crystalline penicillin $G$ lose their antibacterial potency more rapidly than do solutions prepared from partially purified penicillin of somewhat lower purity.

Following clues suggested by the results of ferment. ation experiments ${ }^{1}$, it has been observed repeatedly in these Laboratories that addition of small amounts of phosphate, in the form of suitable mixtures of monobasic and dibasic potassium or sodium phos. phates, markedly retards the destruction of penicillin 